\title{
An algorithm for IoT based vehicle verification system using RFID
}

\author{
Alen Joseph Samuel, Shoney Sebastian \\ Department of Computer Science, CHRIST (Deemed to be University), India
}

\begin{tabular}{l}
\hline Article Info \\
\hline Article history: \\
Received Jan 5, 2019 \\
Revised Apr 8, 2019 \\
Accepted Apr 20, 2019 \\
\hline
\end{tabular}

\section{Keywords:}

Arduino

Identification

Internet of Things

RFID Vehicle

Sensors

\begin{abstract}
The verification of vehicle documents is an important role of transport department which is rising day by day due to the mass registration of the vehicles. An automated vehicle verification system can improve the efficiency of this process. In this paper, we propose an IOT based vehicle verification system using RFID technology. As a result, the vehicle checking which is done now manually can be replaced by automation. There is a loss of a significant amount of time when the normal vehicle checking is done manually. The proposed system will make this process automated. The present verification process is using inductive loops that are placed in a roadbed for detecting vehicles as they pass through the loop of the magnetic field. Similarly, the sensing devices spread along the road can detect passing vehicles through the Bluetooth mechanism. The fixed audio detection devices that can be used to identify the type of vehicles on the road. Other measurements are fixed cameras installed in specific points of roads for categorising the vehicles. But all these mechanisms cannot verify the documents and certificates of the vehicles. In our work, we have suggested an algorithm using RFID technology to automate the documentation verification process of the vehicles like Pollution, Insurance, Rc book etc with the help of RFID reader placed at road checking areas. This documents will be updated by the motor vehicle department at specific periods.
\end{abstract}

Copyright $@ 2019$ Institute of Advanced Engineering and Science. All rights reserved.

\section{Corresponding Author:}

Alen Joseph Samuel,

Department of Computer Science,

CHRIST (Deemed to be University),

Hosur Road, Bangalore, Karnataka, 560029, India.

Email: alen.samuel@cs.christuniversity.in

\section{INTRODUCTION}

Internet of Things or IoT is a concept which connects the systems with the help of embedded technology, the embedded technology is the concept of combining the daily used physical objects with the help of sensors attached, and the information is collected for the future use [1]. The data which is received is from the series of sensors which are connected in a systematic way of obtaining an optimal result. The sensors are used not only to collect data but also to identify and segregate the data [2].

The number of motor vehicle is increasing day by day in roads. The systematic of way of vehicle verification is not happening in streets. Thus the conventional method of vehicle checking is near to impossible in roads nowadays [3]. Due to the increase in the motor vehicle inroads, there is a massive waste of time, and an increase in pollution is happening [4]. Thus the need for vehicle checking done by motor 
vehicle department needs to be done on a regular basis. The customer needs to make sure that the pollution certificate should be up to date [5]. This research is to present an intelligent way of making the routine vehicle checking done inroads to automated [6]. The system is featured by low cost, high performance, easy to maintain functionalities that replace the traditional road checking and the proposed method can improve road checking tremendously [7]. The internet of things is related to sensing the data and communicating it wirelessly. The recognition of objects, tracking them, managing the flow, and monitoring the vehicle is done automatically. The paper proposes an algorithm that relates internet of things with sensors and controls the identification of the vehicle properly and systematically [8]. The heterogeneous data is being classified into different tables, and the segregation of information is being happened with the help of the system [9].

Radio-frequency identification (RFID) is an identification method used to identify and store the information collected with the help of RFID tags or RFID reader [10]. The technology required the use of RFID tag and RFID reader. The technology works very directly; the RFID sensor identifies the RFID tag, and the details which are stored inside the tag can be encrypted with the help of this process. The tracking and identification are done with the help of radio waves [11]. The tag sensor can read the tag from a particular distance beyond the line of sight of the reader. Thus this RFID tag and reader can be used to incorporate into a new product for the tracking and identification using radio waves [12].

\section{RELATED WORK}

Vehicle identification during the time of regular vehicle checking is significant social towards which serious action must be taken. One of the solutions for this is using the Internet of Things which is the current trend in technology. For this purpose, many authors have worked in this domain by applying this technology in their paper.

Celesti et al.,[13] is discussing the condition of the vehicle is monitored using different sensors. The parameters that are addressed in their work are: Inductive loops can be placed in a roadbed for detecting vehicles as they pass through the loop of a magnetic field. Similarly, the sensing devices spread along the road can detect passing vehicles through the Bluetooth mechanism.

Min et al.,[14] is discussing the innovative idea to solve the problem of categorising the heterogeneous data with the help of traffic scheduling delay algorithm. The whole system is of two parts. One computer which is used to allocate communication according to the required traffic data. With the help of IOT large data is being gathered by the wireless sensor network (WSN) and being processed.

Sahadev et al.,[15] is discussing the traffic congestion and monitoring which is getting increased day by day.. The proper way of identifying and monitoring the vehicle will put this scenario. Thus traffic monitoring and controlling have become the biggest challenge in the field of traffic management. This is becoming the main challenge in almost all countries. Most of the metropolitan cities suffer this and related problems.

Talukder et al.,[16] is discussing microcontroller based traffic control system. Using the sensor and live we application the system help to optimise the traffic step by step. The transport infrastructure designed can help the traffic control in cities where the traffic congestion is of great. The paper is discussing the system which used raspberry pie to analyse and find the density of traffic at lanes and cities. With the help of the internet of thing (IOT) the live data is being passed to the server, and live tracking of the traffic is being happened.

Jasmin et al.,[17] is discussing the traffic management system which manages the traffic dynamically with the help of IOT, Infrared sensor and Image processing techniques. The sensor's data which is collected from the IR sensor is then transmitted with the help of the Wifi transmitter which is received by the raspberry controller in the system.

Tärneberg et al.,[18] is discussing the concept of public cloud such as Amazon Web Services (AWS) that can be used for storing the live data. The use of public clouds which supports the connected devices and IoT which connects all this is growing nowadays. The main goal of the research into sense the data with the help sensors and the accuracy of the data is also a goal in the field of research. The scientific experimentation is done with the help of this technology. In this paper, for the need of scientific experiments, the emerging trend of using IOT support in public clouds which will lead to an increase in the productivity is discussed.

Cheng et al.,[19] is discussing in the paper about the various application of RFID tags and RFID sensors. The use of RFID in-vehicle networking is discussed in this paper. The management of the vehicle on the road and its advantage are discussed in the paper. The whole of the system is divided into two parts in this study. The first one is the vehicle access control system and vehicle information control system. The vehicle access control system will control the flow of vehicles and the vehicle passing in and out. 
Cao et al.,[20] is discussing the efficiency parking of vehicles with the help of RFID. The automated technology which is managed in a control centre is being used in this system. The vehicle with RFID can identify, and information can be used to carry out further procedure. The system works in a way that when the RFID vehicle which enters the area is being monitored, and the information of the vehicle is being read using the RFID tag inside the vehicle.

Ying et al.,[21] is discussing the domestic vehicle management and improved design which enable vehicle management system based on RFID technology. The system will enable a safe way of doing all of the tasks. The system is analysing the data based on network communication, remote computer monitoring and card reader technology which enable the vehicle management system.

Marais at al.,[22] is discussing the investigates that is suitability for the UHF which is a passive RFID tag used for monitoring the electronic vehicle identification (EVI) which identify the electric vehicle in the roads. A system is developed so that models of each of the key elements within an EVI environment is being detected. Thus the installation of this will make sure the vehicle is being detected and identified accordingly.

Deng in the paper[23] is discussing the better understanding about IOT operation principle and working mechanism which is combined with the EPCglobal standards which is an IOT structural model which is established. The working modes of the perceptive layer and the network layer are explained. For the further promote and to apply the IOT structure model which is used to control the model.

Jing et al.,[24] is explaining about the speed detection system(SDS) which is based on road surface RFID reader and RFID tag in vehicles. The tag will be on the road all the time. The proposed SDS can be used to detect all road types and full time was speeding detection based on RSR concept.

\section{PROPOSED WORK}

Explaining After considering facts presented, there is a vital need for research in vehicle verification process. The presented vehicle identification and verification system will monitor and track the vehicle in all the conditions. The system contains a server and a display unit for reporting and displaying related events (RFID tagged vehicle details, NON-RFID tagged vehicle details, STOLEN vehicle details).

I'm willing to contribute to the domain-"IOT based vehicle identification system". In this area, many authors have published their work by contributing solutions to this area. The authors Antonio Celesti, Antonino Galletta , Lorenzo Carnevale, Maria Fazio and Aime Lay-Ekuakille [25] have contributed by working on-"An IoT Cloud System for Traffic Monitoring and Vehicular Accidents Prevention Based on Mobile Sensor Data Processing". Following is the block diagram for the system as shown in Figure 1.

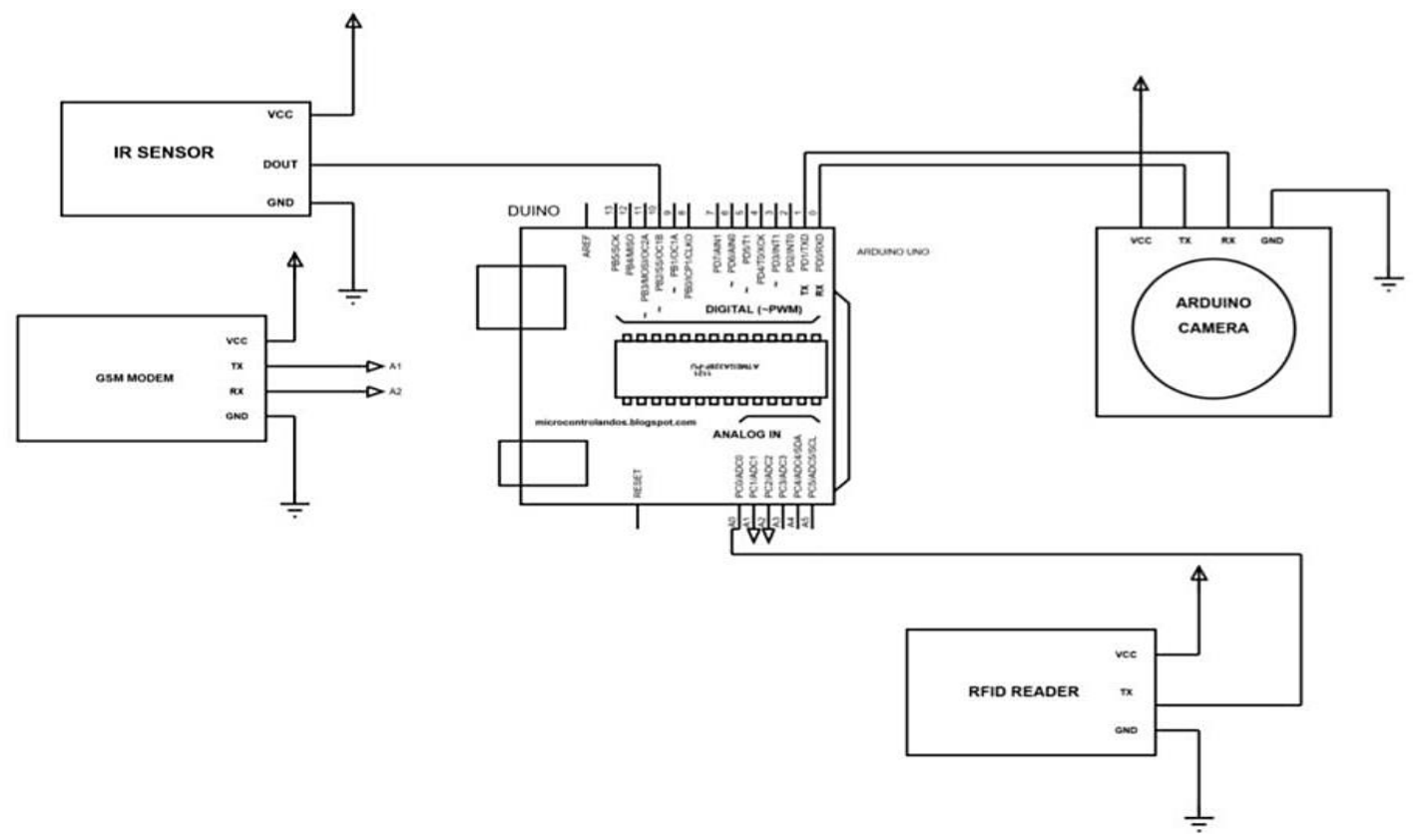

Figure 1. Block diagram of proposed system 


\subsection{Target of automatic vehicle identification system \\ a. Vehicle certificate checking}

When the vehicle moves through the RF reader, the RF Reader reader will read the vehicle ID by scanning the RF tag, and the associated module will validate the vehicle ID with the pre-stored records in the database and automatically checking for all certificates validity. If any invalid details are found, the system will make an alert to the motor department via email. Thus the department can take further actions on the system from the generated report. Table 1 shows the mandatory certificate details template of each RFID tags.

Table 1. Vehicle certificate details template

\begin{tabular}{lll}
\hline RC Book & PUC Certificates & Insurance Certificate \\
\hline Owner Name & Vehicle Name & Company Name \\
Engine Number & PUC Number & Insurance Id \\
Reg Number & Model Number & Insurance Type \\
Issue Date & Issue Date & Issue Date \\
Expiry Date & Expiry Date & Expiry Date \\
\hline
\end{tabular}

Rc Book: It is the Registration Certificate of the vehicle which allows the vehicle to be legally drive in the roads.

PUC Certificate: Pollution Under Control certificate gives information about the emotion of gas and standards by the government.

Insurance Certificate: As per rule 141 of Act, every vehicle should have an insurance certificate with a valid insurance cover.

\section{b. Normal vehicle}

When the normal vehicle with RFID tag passes before the IR sensor, it is detected after that a count of 5 seconds is initiated before the 5 seconds the vehicle has to pass the RFID reader, which it will as both are placed nearby. When the normal card is read the RFID sequence number is taken, and the vehicle can be identified as a normal vehicle, and the output can be seen as "Normal vehicle."

\section{c. Stolen vehicle}

A stolen vehicle was initially a normal vehicle when it was first registered it becomes stolen once it is missing or suspected to be stolen, at that situation the owner approaches and designate his vehicle as stolen one now the RFID card number corresponding to the registration no: will be now designated as stolen.

E.g.: During registration:

K1 01 AA $1234=X($ a tag code $)=$ Normal Vehicle

When it is identified as a stolen vehicle;

KL 01 AA $1234=\mathrm{X}$ (same tag code as before $)=$ Stolen Vehicle

Now the vehicle is set as stolen and whenever this vehicle is read, it will be identified as a stolen vehicle. At the instant of detecting a stolen vehicle, a message will be sent to the concerned personals number with the help of the GSM module that the vehicle.

\section{d. Vehicle without RFID tag}

For a vehicle without RFID tag, As the vehicle passes the IR sensor it will be detected and the count of 5 seconds will be initiated, but as there is no RFID tag the vehicle will not be read, and the display shows "Vehicle not registered". At this instant, a low pulse signal will be sent to the pi module from the Arduino. A pi camera is connected to the pi module which requires a low pulse for operation. So as now the vehicle is detected the low pulse will be transmitted and the camera will be triggered, and the image of the car will be taken. So, this image can be analyzed by the operating person and along with the owner of the vehicle and by analyzing the physical features the owner can find the car and hence find the last seen location.

\subsection{Proposed algorithm}

The main functioning behind the proposed system is the detect and identify the vehicles algorithm that takes different inputs into account and based on that it generates results that are helpful for determining the status of the proposed system. 


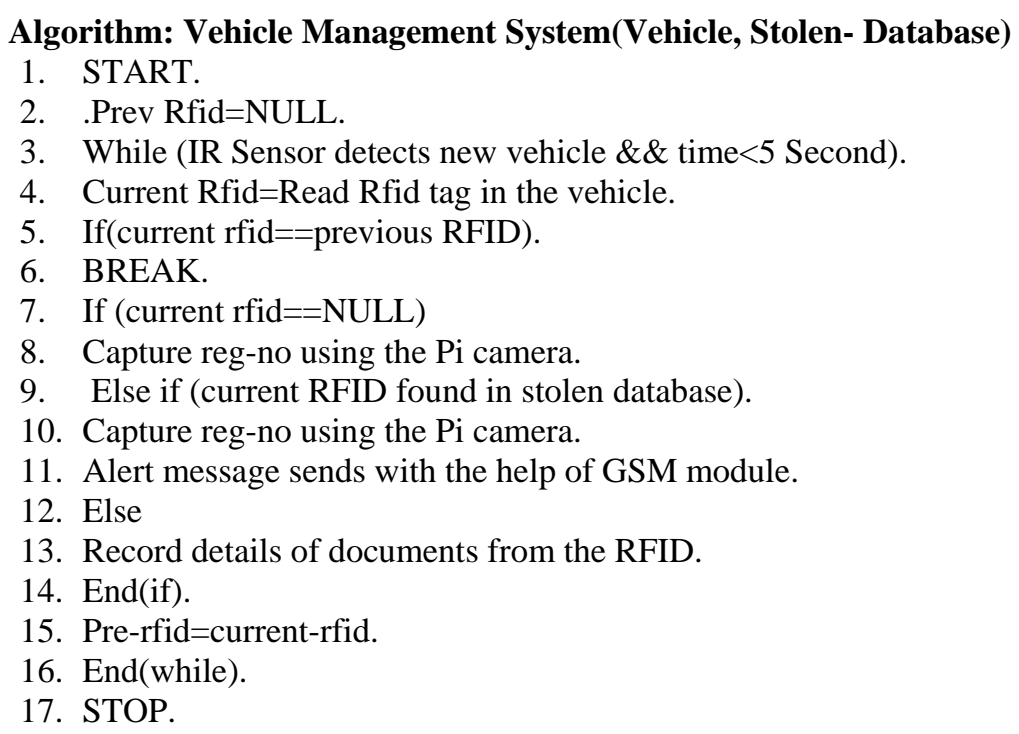

\section{RESULTS AND ANALYSIS}

In The system is simulated using Arduino IDE as a tool to generate test results for each sensor by giving an input value to it. To use this tool, it is required to use a programmable circuit commonly known as a microcontroller which is the main component for this tool used. The code is written for sensors in $\mathrm{C}$ programming language in Arduino IDE and flashed to the microcontroller to test the sensor. The data generated by the sensor can be analyzed in the output screen of the Arduino IDE.

\subsection{Stimulation for checking vehicle certificates}

Table 2 represents the details of vehicle certificates; it checks whether the certificates are present with the owner of the vehicle or not.When the vehicle moves through the RF reader, the RF Reader reader will read the vehicle ID by scanning the RF tag, and the associated module will validate the vehicle ID with the pre-stored records in the database and automatically checking for all certificates validity. If any invalid details are found, the system will make an alert to the motor department via email. Thus the department can take further actions on the system from the generated report.

Table 2. Vehicle certificate dataset

\begin{tabular}{llcl}
\hline Vehicle ID & RC Book & PUC Certificates & Insurance Certificate \\
\hline BH40 & YES & YES & YES \\
BH41 & YES & NO & NO \\
BH42 & NO & YES & YES \\
BH43 & NO & NO & NO \\
BH44 & YES & YES & YES \\
BH45 & YES & YES & NO \\
\hline
\end{tabular}

Figure 2 represents the graph between the Vehicle ID and the Vehicle certificate status. According to the graph, the vehicle certificate status can be found out, and further measures can be taken to let the customer about the missing certificates. Note that Red dots indicate PUC certificate, Blue indicates RC book, and Green indicates Insurance certificate. 


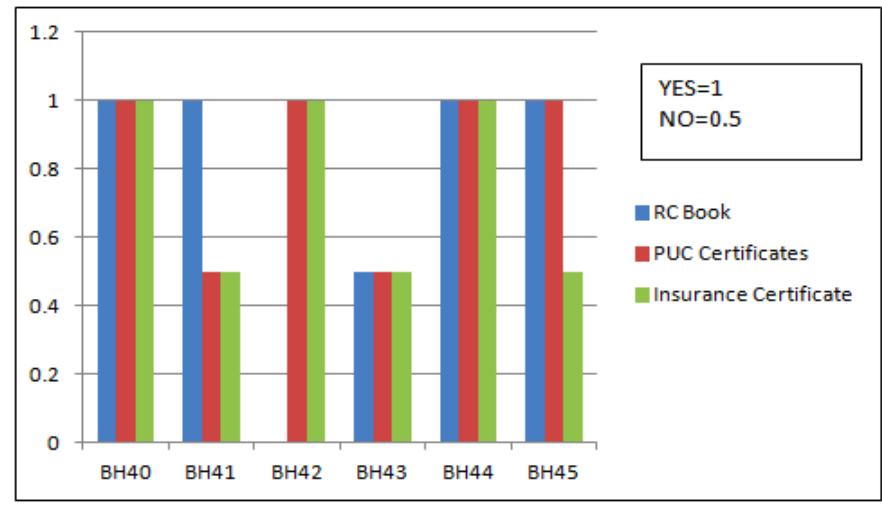

Figure 2. Graph between Vehicle ID and Certificate status

\subsection{Stimulation for RFID based vehicle identification system}

Table 3 shows the sample dataset information which is collected from the RFID reader. The RFID card number and corresponding RFID reader data are being shown in the table. The system also provides the time with the RFID reader identified the RFID card data, and the status of the data is also being identified.

Table 3. RFID Reader Dataset

\begin{tabular}{clrl}
\hline RFID Card & RFID Reader & \multicolumn{1}{l}{ Time } & Status \\
\hline 101 & 1010 & $9: 00$ & With RFID \\
102 & 1011 & $9: 15$ & Without RFID \\
103 & 1012 & $10: 00$ & With RFID \\
104 & 1013 & $10: 15$ & Without RFID \\
105 & 1014 & $11: 00$ & Without RFID \\
106 & 1015 & $11: 15$ & With RFID \\
107 & 1016 & $12: 00$ & STOLEN \\
108 & 1017 & $12: 15$ & Without RFID \\
109 & 1018 & $13: 00$ & With RFID \\
110 & 1019 & $13: 15$ & With RFID \\
\hline
\end{tabular}

Figure 3 represents the graph between the status of the vehicle and the RFID tag. According to the graph, When an RFID tagged the vehicle, a non-RFID tagged vehicle and a Stolen vehicle is passed through the RFID reader it categorize the data into RFID tagged vehicle, Non-RFID tagged vehicle and Stolen Vehicle accordingly. Note that Red dots indicate Stolen vehicle, Blue indicates without RFID vehicle, and Green indicated with RFID vehicle.

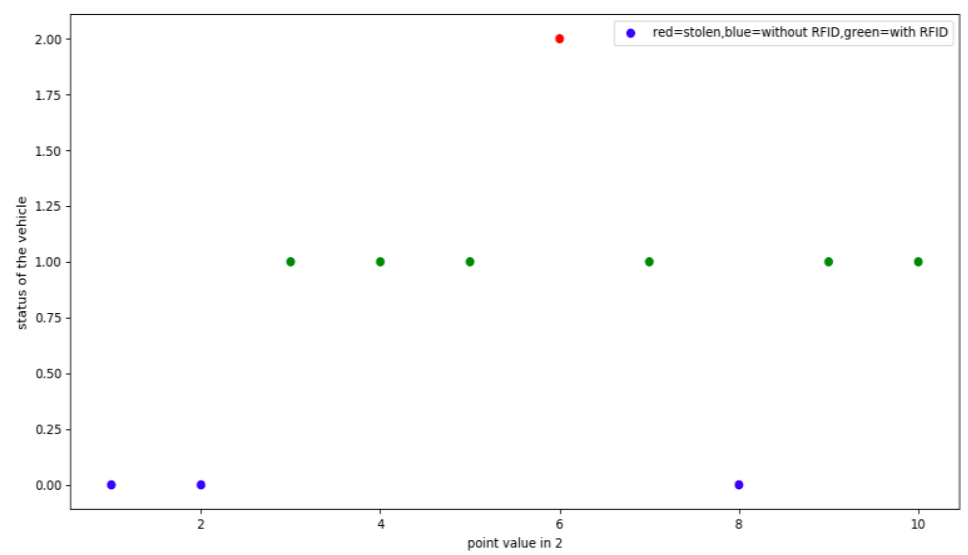

Figure 3. Graph showing status of the vehicle 


\section{CONCLUSION}

The system is designed as to automate the -road vehicle checking which is done by the motor department. For which we are introducing a technique in which every vehicle should have RF Device which had an RFID tag fitted with the vehicle during the time of buying the vehicle. By replacing the on-road checking done by motor department, the RF Reading device placed near by the road will read the card which is placed in vehicles and automatically identifies the owner details and corresponding certificate which need to fetch the details from. The department can track if there is any mismatch in the vehicle certificates. The vehicle which does not have RFID tag can also be found with the help of this technique. Another facility provided by the system is stolen vehicle detection in the system. The lost vehicle can be found when the vehicle is being passed through any of the RFID readers in the roads. If the robber removes the tag from the vehicle, also the vehicle is identified as a NON-RFID tagged vehicle and the vehicle can be found out when motor department contacts the customer for installing RFID tag in the vehicle. Thus the system provides an easy and systemic way if vehicle identification and management.

\section{REFERENCES}

[1] Upendra Yadav, Kamalakannan, "Smart Vehicle Monitoring System using IOT," International Journal for Development of Computer Science and Technology, Vol-5,I-3,SW-31

[2] Pallavi A Targe and M P Satone "VANET based Real-Time Intelligent Transportation System" International Journal of Computer Applications, Vol 145 - No.4, July 2016.

[3] Dhanlakshmi and Ezil Sam Leni, "Instance Vehicle Monitoring and Tracking with Internet of Things using Arduino," International Journal on Smart Sensing and Intelligent Systems, September 2017.

[4] Ahmad, R. M. Noor, I. Ali, M. Imran, and A. Vasilakos, "Characterizing the role of vehicular cloud computing in road traffic management," Int. J. Distrib. Sensor Netw., vol. 13, pp. 1-14, Apr. 2017.

[5] F. Lin, Q. Huang, and Q. Tan, "Distributed storage video cloud of intelligent transportation system based on the backward cloud generator," Adv. Transp. Stud., vol. 2, pp. 15-22, 2015.

[6] Dong-Liang Wu et al., "A brief survey on current RFID applications," 2009 International Conference on Machine Learning and Cybernetics, Vol.4, pp.2330-2335, July 2009.

[7] Soichi Kubota et al., "Safety Driving Support System Using RFID for Prevention of Pedestrianinvolved Accidents," 2006 6th International Conference on ITS Telecommunications Proceedings, pp. 226-229, 2006.

[8] Wang Hongjian et al., "RFID Technology Applied to Monitor Vehicle in Highway," 2012 Third International Conference on Digital Manufacturing \& Automation, pp.736-739, July 2012.

[9] Kuo-Shien Huang et al., "RFID Applications Strategy and Deployment in Bike Renting System," 10th International Conference on Advanced Communication Technology, Feb. 2008, Vol.1, pp. 660-663, 2008.

[10] Pandit, A. A et al., "RFID Tracking System for Vehicles (RTSV)," First International Conference on Computational Intelligence, Communication Systems and Networks, July 2009, pp.160-165, 2009.

[11] M. V. Bueno-Delgado et al., "A geometric distribution reader anti-collision protocol for RFID dense reader environments,” IEEE Trans. Autom. Sci. Eng., vol. 10, no. 2, pp. 296-306, Apr. 2013.

[12] H. Landaluce at el., "An energy and identification time decreasing procedure for memoryless RFID tag anti collision protocols," IEEE Trans. Wireless Commun., vol. 15, no. 6, pp. 4234-4247, Jun. 2016.

[13] Antonio Celesti et al., "An IoT Cloud System for Traffic Monitoring and Vehicular Accidents Prevention Based on Mobile Sensor Data Processing," IEEE Sensors Journal, vol. 18, no. 12, June 15, 2018.

[14] Minghui Min et al., "Traffic Aware Multiple Slot Frames Scheduling Algorithm in Industrial loT Applications Using IEEE S02.1S.4e TSCH," IEEE 16th International Conference on Communication Technology (ICCT), 2015.

[15] Varsha Sahadev et al., "An Intelligent Framework for Vehicle Traffic Monitoring System using IoT," International Conference on Intelligent Computing and Control (I2C2), 2017.

[16] Mehal Zaman Talukder et al., "An IoT Based Automated Traffic Control System With Real-Time Update Capability," 8th International Conference on Computing, Communication and Networking Technologies (ICCCNT), 2017.

[17] Paul Jasmine Rani et al., "Dynamic traffic management system using infrared (ir) and internet of things (iot)," Third International Conference on Science Technology Engineering \& Management (ICONSTEM), 2017.

[18] William Tärneberg et al., "Experiences Creating a Framework for Smart Traffic Control using AW IOT," IEEE/ACM 9th International Conference on Utility and Cloud Computing, 2016. 
[19] Xiaolu Cheng et al., "A Survey on RFID Applications in Vehicle Networks," International Conference on Identification, Information, and Knowledge in the Internet of Things (IIKI), 2015.

[20] Zijuan Cao et al., "A Vehicle Management System of Community Based on Radio Frequency Identification Technology," IEEE 3rd International Conference on Communication Software and Networks, 2011.

[21] Jiang Lin-ying et al., "Improved Design of Vehicle Management System Based on RFID," International Conference on Intelligent System Design and Engineering Application, 2010.

[22] H. Marais et al., "Modelling of an RFID-based Electronic Vehicle Identification System," M.engdissertation, North-West University, South Africa, 2008.

[23] Na Deng "RFID Technology and Network Construction in the Internet of Things," International Conference on Computer Science and Service System, 2012.

[24] Tao Jing et al., "Speeding Detection in RFID Systems on Roads," International Conference on Connected Vehicles and Expo (ICCVE), 2013.

[25] Antonio Celesti et al., "An IoT Cloud System for Traffic Monitoring and Vehicular Accidents Prevention Based on Mobile Sensor Data Processing," IEEE Sensors Journal, Volume: 18, Issue: 12, June 15, 2018.

\section{BIOGRAPHIES OF AUTHORS}

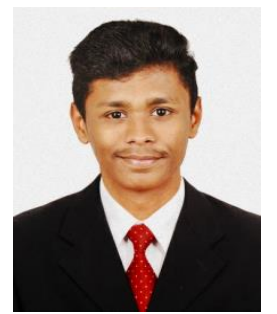

Alen Joseph Samuel pursuing the Msc Computer Science post graduation degree from CHRIST (Deemed to be University), India. He had received degree from CHRIST (Deemed to be University).His research interest is iot based vehicle identification system using RFID.

E-mail: alen.samuel@cs.christuniversity.in

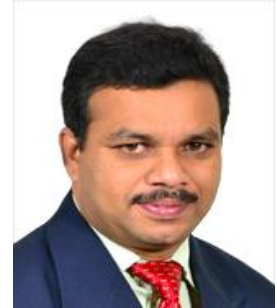

Shoney Sebastian received MPhil degree from Vinayaka Missions University in 2007.MCA degree from Indira Gandhi National Open University in 2000.PGDCA degree from Institute of Human Resource Development for Electronics in 1995 and now pursuing $\mathrm{PhD}$ degree from CHRIST (Deemed to be University).Currently, he is working as the Associate Professor in CHRIST (Deemed to be University).His research interest includes cloud computing, data mining and Indoor Positioning system.

E-mail:shoney.sebastian@christuniversity.in 